\title{
DUALITY OF CONSERVATION LAWS AND THEIR ROLE IN THE PROCESSES OF EMERGENCE OF PHYSICAL STRUCTURES AND FORMATIONS
}

\author{
LUDMILA I. PETROVA
}

\begin{abstract}
As it is known, the conservation laws for material media are conservation laws for energy, linear momentum, angular momentum, and mass. Such conservation laws are described by differential equations. And the conservation laws for physical fields are conservation laws that state the presence of conservative physical quantities or objects (structures). Such conservation laws are described by closed exterior skew-symmetric forms. It can be seen that conservation laws possess duality. The conservation laws for material media and the conservation laws for physical fields are different. A peculiarity consists in the fact that there exists a connection between the conservation laws for material media and those for physical fields. This connection is realized discretely in the evolutionary process. It describes the emergence of physical structures and the observed formations, such as waves, vortices, turbulent pulsations.
\end{abstract}

\section{INTRODUCTION}

What meaning does the concept of "conservation laws" contain? In the process of scientific development, the concept of "conservation laws" in thermodynamics, physics and mechanics assumed a different sense.

In thermodynamics, the conservation laws are associated with the principles of thermodynamics. The first principle of thermodynamics that relates to the energy conservation law can be written as [1]

$$
d E+d w=\delta Q
$$

where $d E$ is the energy variation, $d w$ is the work done by the system, $\delta Q$ is the heat delivered to the system. (Here, an ambiguity is observed; this relation includes not only energy, but the mechanical work as well. This ambiguity of the first thermodynamic principle and the peculiarity of the second thermodynamic principle will be explained below.)

In the mechanics and physics of continuous media the concept of "conservation laws" relates to the conservation laws for energy, linear momentum, angular momentum, and mass that establish the balance between the variations of physical quantities and external action. These are conservation laws that are described

MSC (2010): primary 35A70, 58J70, $70 \mathrm{H} 33$.

Keywords: the conservation laws for material media, the conservation laws for physical fields, skew-symmetric forms, nonintegrable manifold, physical structures and the observed formations. 
by differential equations. (From here on, they will be referred to as the balance conservation laws.)

In areas of physics related to the field theory and in the theoretical mechanics "the conservation laws" are those according to which the conservative physical quantities or objects exist. These are conservation laws that are described by closed exterior forms. (Below, they will be referred to as "exact" ones.) The Noether theorems can serve as an example of such conservation laws formulation, which, under some conditions, can be written as

$$
d \omega=0
$$

where $\omega$ is an exterior skew-symmetric differential form. (It is of interest to call attention to the fact that formulas (1.1), (1.2) have the form of relations in skewsymmetric differential forms.)

Thus, the concept of "the conservation laws" relates to the balance conservation laws, exact conservation laws and the principles of thermodynamics.

Now the question arises whether there is any connection between them.

The mathematical apparatus of the skew-symmetric differential forms allows us to answer this question [2-5].

It will be shown that the balance and exact conservation laws are related to each other. The exact conservation laws are obtained from the balance (differential) conservation laws as a result of interactions among the balance conservation laws (which appear to be noncommutative).

The principles of thermodynamics are just examples of considering such interaction (mutual influence). The principles of thermodynamics integrate two balance conservation laws, namely, the balance conservation law for energy and that for linear momentum.

The duality of conservation laws plays a regulating role in the evolutionary processes taking place in material media. Transitions from equations corresponding to balance conservation laws to closed external forms corresponding to exact conservation laws describe transitions of the state of material medium from a nonequilibrium state to a local equilibrium state, which is accompanied by the emergence of physical structures and the appearance of observable formations such as fluctuations, waves, turbulent pulsations and so on.

The results of the present paper are obtained with the help of skew-symmetric differential forms whose properties correspond to conservation laws. Besides the exterior forms $[2,3,5]$, the skew-symmetric forms, whose basis, as opposed to exterior forms, are nonintegrable manifolds, are used [3,4]. Mathematical apparatus of such skew-symmetric forms, which possess the evolutionary properties, includes nontraditional elements like nonidentical relations and degenerate transformations and this enables one to describe evolutionary processes and generation of various structures. Such skew-symmetric differential forms possess a unique property, namely, they can generate closed exterior forms that are invariants and correspond to the exact conservation laws. 


\section{EXACT CONSERVATION LAWS}

The exact conservation laws are those that state the existence of conservative physical quantities or conservative physical objects. The conservation laws for physical fields are such conservation laws.

The closed exterior skew-symmetric differential forms correspond to the exact conservation laws $[2-5]$.

\subsection{Some properties of skew-symmetric forms corresponding to the exact conservation laws}

Closed inexact exterior forms. The external differential form of the degree $p$ ( $p$-form) can be written as $[4,5]$ :

$$
\theta^{p}=\sum_{i_{1} \ldots i_{p}} a_{i_{1} \ldots i_{p}} d x^{i_{1}} \wedge d x^{i_{2}} \wedge \cdots \wedge d x^{i_{p}} \quad 0 \leq p \leq n .
$$

Here, $a_{i_{1} \ldots i_{p}}$ is the function of independent variables $x^{1}, \ldots, x^{n}, n$ is the space dimension, and $d x^{i}, d x^{i} \wedge d x^{j}, d x^{i} \wedge d x^{j} \wedge d x^{k}, \ldots$ is the local basis subject to the condition of skew-symmetry:

$$
\begin{aligned}
& d x^{i} \wedge d x^{i}=0, \\
& d x^{i} \wedge d x^{j}=-d x^{j} \wedge d x^{i} \quad i \neq j .
\end{aligned}
$$

The exterior form differential $d \theta^{p}$ is expressed by the formula

$$
d \theta^{p}=\sum_{i_{1} \ldots i_{p}} d a_{i_{1} \ldots i_{p}} \wedge d x^{i_{1}} \wedge d x^{i_{2}} \cdots \wedge d x^{i_{p}} .
$$

There is no differential of the basis since the basis is integrable manifolds or structures.

The skew-symmetric form is called a closed one if its differential equals zero:

$$
d \theta^{p}=0 .
$$

From condition (2.1) one can see that the closed form is a conservative physical quantity. This means that such a form can correspond to the exact conservation law.

If the form is closed only on a pseudostructure, i.e., this form is a closed inexact one, the closure condition can be written as

$$
d_{\pi} \theta^{p}=0 .
$$

In this case, the pseudostructure $\pi$ obeys the condition

$$
d_{\pi}^{*} \theta^{p}=0
$$

here, ${ }^{*} \theta^{p}$ is the dual form. ${ }^{1}$

From conditions (2.2) and (2.3) one can see that the dual form (pseudostructure) and the closed inexact exterior form (conservative physical quantity) describe a conservative physical object that can also correspond to the exact conservation law.

\footnotetext{
${ }^{1}$ As will be shown below, closed exterior forms are defined on a cotangent (covariant) manifold. Pseudostructures are the bundle of a cotangent manifold.
} 
The closed inexact exterior and dual forms form a differential-geometrical structure that describes a structure with a conservative physical quantity.

The physical structures, i.e., pseudostructures with a conservative physical quantity, are conservative physical objects. Thats is, they are subject to conservation laws for physical fields.

It is evident that the exact conservation law is one for physical fields. The closed form is a differential of a form of lower degree: $\theta^{p}=d \theta^{p-1}$. In this case, the closedness condition is satisfied: $d \theta^{p}=d d \theta^{p-1}=0$. A closed inexact form is a differential too; and, in this case, the differential is an interior one defined on a pseudostructure.

Since a closed form is a differential, then it is obvious that a closed form will turn out to be invariant under all transformations that conserve the differential. The nondegenerate transformations in mathematics and mathematical physics such as the unitary, tangent, canonical, gradient, and other nondegenerate transformations are examples of such transformations that conserve the differential.

It is obvious that conservative physical quantities and conservative physical structures, which are obtained from closed external forms, are physical quantities and physical structures that are conserved in nondegenerate transformations.

As it will be shown below, the closed inexact exterior forms, which describe exact conservation laws corresponding to conservation laws for physical fields, are obtained from the equations of the balance (differential) conservation law for the material media.

\section{BALANCE CONSERVATION LAWS}

The balance (differential) conservation laws are the conservation laws for energy, linear momentum, angular momentum, and mass. These are the conservation laws for the material media (material systems), such as thermodynamic, gas dynamical, cosmic systems, and others. Such conservation laws establish the balance between the change of the physical quantities of material medium and external actions.

The balance conservation laws are described by differential equations for energy, linear momentum, angular momentum, and mass. The equations of mathematical physics for material media consist of the equations of the balance conservation laws $[6-8]$.

With the help of the skew-symmetric differential forms one can see (even without knowledge of the concrete form of equations of mathematical physics) specific features of these equations that elucidate the properties of the balance conservation laws. To do so it is necessary to study the consistency of equations of the balance conservation laws.

\subsection{Analysis of balance conservation law equations for material media. Evolutionary relation}

A peculiarity of the present investigation is that the conservation law equations are transformed into equations expressed in terms of state functionals.

Equations of conservation laws for material media can be written for state functionals. Since the physical quantities (like temperature, energy, pressure, density) relate to a single material medium, a connection between them should exist. Such 
a connection is described by state functionals. The functionals such as wave function, entropy, the action functional, the Pointing tensor, the Einstein tensor and so on, which are the field-theory functionals, are also functionals of the equations describing material media [9].

Let us analyze the equations that describe the balance conservation laws for energy and linear momentum $[6,8]$.

The consistency of equations can only be investigated using two frames of reference.

We introduce two frames of reference: the first is an inertial one (this frame of reference is not connected with the material medium), and the second is an accompanying one (this frame of reference is connected with the manifold constructed of the trajectories of the material medium elements). The Euler and Lagrange frames of reference are examples of such frames.

The energy equation in the inertial frame of reference can be reduced to the form:

$$
\frac{D \psi}{D t}=A_{1}
$$

where $D / D t$ is the total derivative with respect to time, $\psi$ is the functional of the state that specifies the material medium, $A_{1}$ is the quantity that depends on specific features of the medium and on external energy actions onto the medium. ${ }^{2}$

In the accompanying frame of reference, the total derivative with respect to time is transformed into the derivative along the trajectory. Equation (3.1) is now written in the form

$$
\frac{\partial \psi}{\partial \xi^{1}}=A_{1}
$$

here, $\xi^{1}$ is the coordinate along the trajectory.

In a similar manner, in the accompanying frame of reference the equation for linear momentum is reduced to the equation of the form

$$
\frac{\partial \psi}{\partial \xi^{\nu}}=A_{\nu}, \quad \nu=2, \ldots
$$

where $\xi^{\nu}$ are the coordinates in the direction normal to the trajectory, $A_{\nu}$ are the quantities that depend on the specific features of the medium and external force actions. Equations (3.2), (3.3) can be convoluted into the relation

$$
d \psi=A_{\mu} d \xi^{\mu}, \quad(\mu=1, \nu)
$$

where $d \psi$ is the differential expression $d \psi=\left(\partial \psi / \partial \xi^{\mu}\right) d \xi^{\mu}$. Relation (3.4) can be written as

$$
d \psi=\omega
$$

Here, $\omega=A_{\mu} d \xi^{\mu}$ is the skew-symmetric differential differential form of the first degree.

\footnotetext{
${ }^{2}$ The action functional, entropy, wave function can be regarded as examples of the functional $\psi$. Thus, the equation for energy presented in terms of the action functional $S$ has a similar form: $D S / D t=L$, where $\psi=S, A_{1}=L$ is the Lagrange function. In mechanics of continuous media, the equation for energy of ideal gas can be presented in the form: $D s / D t=0$, where $s$ is entropy. In this case, $\psi=s, A_{1}=0$.
} 
Relation (3.5) was obtained from the equation of the balance conservation laws for energy and linear momentum. In this relation the form $\omega$ is that of the first degree. If the equations of the balance conservation laws for angular momentum are added to the equations for energy and linear momentum, this form in the evolutionary relation will be the form of the second degree. And in combination with the equation of the balance conservation law of mass this form will be the form of degree 3 .

Thus, in the general case, the evolutionary relation can be written as

$$
d \psi=\omega^{p}
$$

where the form of degree $p$ takes the values $p=0,1,2,3$. (The evolutionary relation for $p=0$ is similar to that in the skew-symmetric differential forms, and it was obtained from the interaction of energy and time.)

The relations obtained and the skew-symmetric forms $\omega^{p}$ are evolutionary ones since the conservation law equations are evolutionary ones.

In the Appendix, the derivation of the evolutionary relation for a gas-dynamic medium is given.

The evolutionary relation possesses nontraditional mathematical apparatus that includes such elements as degenerate transformations, non-identity, and self-change. This is due to the properties of the evolutionary skew-symmetric form [4].

\subsection{Distinction of evolutionary skew-symmetric differential forms from exterior skew-symmetric differential forms}

The evolutionary skew-symmetric differential forms are obtained from differential equations that describe evolutionary processes in material, informational and other open systems [4].

In contrast to exterior forms, they are defined on the deforming nonintegrable manifolds, such as Lagrangian manifold, an accompanying manifold (manifold formed by the trajectories of particles of the described system), etc.

The specific feature of skew-symmetric forms is related to the properties of nonintegrable manifolds [10] (see "Mathematical additions").

The evolutionary form can be written in a manner similar for the exterior differential form [4]. However, in distinction from the exterior form differential, an additional term will appear in the evolutionary form differential. This is due to the fact that the evolutionary form basis changes since such a form is defined on a nonintegrable manifold $[4,10]$.

The evolutionary form differential takes the form

$$
d \theta^{p}=\sum_{i_{1} \ldots \beta_{p}} d a_{i_{1} \ldots B_{p}} \wedge d x^{i_{1}} \wedge d x^{i_{2}} \cdots \wedge d x^{i_{p}}+\sum_{i_{1} \ldots \beta_{p}} a_{i_{1} \ldots \beta_{p}} d\left(d x^{i_{1}} \wedge d x^{i_{2}} \cdots \wedge d x^{i_{p}}\right)
$$

where the second term relates to the basis differential being nonzero:

$$
d\left(d x^{i_{1}} \wedge d x^{i_{2}} \wedge \cdots \wedge d x^{i_{p}}\right) \neq 0 .
$$

(For the exterior form defined on an integrable manifold one has $d\left(d x^{i_{1}} \wedge d x^{i_{2}} \wedge\right.$ $\left.\cdots \wedge d x^{i_{p}}\right)=0$.)

The peculiarity of skew-symmetric forms defined on a nonintegrable manifold can be demonstrated by the example of a skew-symmetric form of first-degree. Let 
us consider the first-degree form $\omega=a_{\alpha} d x^{\alpha}$. The differential of this form can be written as $d \omega=K_{\alpha \beta} d x^{\alpha} d x^{\beta}$, where $K_{\alpha \beta}=a_{\beta ; \alpha}-a_{\alpha ; \beta}$ are components of the commutator of the form $\omega$, and $a_{\beta ; \alpha}, a_{\alpha ; \beta}$ are covariant derivatives. If we express the covariant derivatives in terms of connectedness (if it is possible), they can be written as $a_{\beta ; \alpha}=\partial a_{\beta} / \partial x^{\alpha}+\Gamma_{\beta \alpha}^{\sigma} a_{\sigma}$, where the first term results from differentiating the form coefficients, and the second term results from differentiating the basis. If we substitute the expressions for covariant derivatives into the formula for commutator components, we obtain the following expression for commutator components of the form $\omega$ :

$$
K_{\alpha \beta}=\left(\frac{\partial a_{\beta}}{\partial x^{\alpha}}-\frac{\partial a_{\alpha}}{\partial x^{\beta}}\right)+\left(\Gamma_{\beta \alpha}^{\sigma}-\Gamma_{\alpha \beta}^{\sigma}\right) a_{\sigma} .
$$

Here, the expressions $\left(\Gamma_{\beta \alpha}^{\sigma}-\Gamma_{\alpha \beta}^{\sigma}\right)$ entered into the second term are just components of the commutator of the first-degree metric form that specifies the manifold deformation and hence is nonzero $[4,10]$. (It is well known that the metric form commutators of the first-, second- and third degrees specifies, respectively, torsion, rotation, and curvature.)

In the commutator of exterior form, which is defined on an integrable manifold, the second term is absent: the connectednesses are symmetric, that is, the expression $\left(\Gamma_{\beta \alpha}^{\sigma}-\Gamma_{\alpha \beta}^{\sigma}\right)$ vanishes.

Since the commutator, and hence the differential, of a skew-symmetric form defined on a nonintegrable manifold are nonzero, this means that such a form cannot be a closed one.

The skew-symmetric form, which is obtained from the conservation law equations for material media, is just such an evolutionary form. The basis of this form is an accompanying manifold, namely, the manifold built by the trajectories of the material medium elements. Such a manifold is a deforming nonintegrable manifold. This indicates that the skew-symmetric differential form, which is on the right-hand side of the evolutionary relation, is not a closed form (its differential is not equal to zero).

\subsection{Non-identity and self-change of the evolutionary relation. Non- commutativity of the balance conservation laws.}

Let us show that the evolutionary relation obtained from the equations of the balance conservation laws turns out to be nonidentical.

The skew-symmetric differential form which is on the right-hand side of the evolutionary relation is not a closed form and cannot be a differential like the left-hand side. To do so, we shall analyze relation (3.5).

A relation can be an identical one if this is a relation between measurable (invariant) quantities or between observable (metric) objects. In other words, between quantities or objects that are comparable.

On the left-hand side of evolutionary relation (3.5) there is a differential that is a closed form. This form is an invariant object. The skew-symmetric differential form which is on the right-hand side of the evolutionary relation is not a closed form and cannot be a differential, i.e., invariant like the left-hand side. 
This evolutionary relation is a nonidentical one as it involves an unclosed differential form. ${ }^{3}$

An example of a non-identical evolutionary relation is the first principle of thermodynamics, which consists of the conservation laws of energy and momentum (mechanical work).

The evolutionary relation possesses one more peculiarity, namely, this relation is a self-changing relation. Since one of the objects is an immeasurable quantity, the other cannot be compared with the first one, and hence, the process of mutual variation cannot stop.

The properties of the evolutionary skew-symmetric differential forms and features of the nonidentical evolutionary relation obtained from the balance conservation laws equations allow us to elucidate the specific features of the balance conservation laws and their role in evolutionary processes.

\section{ROLE OF THE BALANCE CONSERVATION LAWS IN EVOLUTIONARY PROCESSES}

It was shown that the evolutionary relation obtained from the balance (differential) conservation law equations proves to be nonidentical. This means that the balance conservation law equations are not consistent.

The inconsistency of the balance conservation law equations indicates that the balance conservation laws are not commutative. To what result does the noncommutativity of balance conservation laws lead? Noncommutativity of the balance conservation laws reflects the state of material medium.

Let us consider evolutionary relation (3.6). On the left-hand side of the evolutionary relation there is a differential $d \psi$ of functional $\psi$ that specifies a state of material medium. Availability of the differential of functional means that there exists a state function, and this indicates equilibrium state of material medium. But since evolutionary relation turns out to be nonidentical, from evolutionary relation it is impossible to obtain the differential of functional. This indicates an absence of the state function and means that the material medium is in non-equilibrium state.

The nonequilibrium state of the material medium means that there is an internal force in the material medium. It is evident that the internal force originates at the expense of some quantity described by the evolutionary form commutator. (If the evolutionary form commutator is zero, the evolutionary relation would be identical, and this would point to the equilibrium state, i.e., the absence of internal forces.) Everything that gives a contribution into the evolutionary form commutator leads to the emergence of the internal force.

Here, attention should be drawn to some properties of the physical quantities of the material medium.

\footnotetext{
${ }^{3}$ As shown, the skew-symmetric form, which enters the evolutionary relation, is defined on an accompanying manifold, namely, the manifold built by the trajectories of the material medium elements. Such a manifold is a deforming nonintegrable manifold. The differential form defined on such manifold cannot be closed, and the corresponding evolutionary relation cannot be identical. A nonidentical relation was analyzed in the paper by J. L. Synge "Tensorial Methods in Dynamics" (1936). And yet it was allowed a possibility to use the sign of equality in a nonidentical relation.
} 
External actions in real processes are not potential impacts. Their nature is different from that of the material medium itself. For this reason, they cannot directly go into the physical quantities of the material medium itself. (Here we mean actions external to the local area of the material medium. The local area of the material medium can be formed by an element of the material medium and its surroundings [2]. Internal force is the force acting inside the local area of the material medium.)

The noncommutativity of the balance conservation laws does not allow a direct transformation of the external actions into the physical quantities of the material medium. The changed quantities are inconsistent and cannot become physical quantities of the material medium. As a result, there arises an unmeasurable quantity that is described by the commutator of the evolutionary form and acts as an internal force.

Can the material media get rid of the internal force and transfer into the equilibrium state? The equilibrium state of the material medium corresponds to the availability of the differential $d \psi$ of functional $\psi$ that specifies a state of material medium. (Availability of the differential of functional means that there exists a state function, and this indicates the equilibrium state of the material medium.) But, as noted above, we cannot get the differential $d \psi$ from a nonidentical relation.

However, under degenerate transform, the relation, which is identical on the pseudostructure, can be obtained from the nonidentical evolutionary relation. From such a relation the differential of a functional on a pseudostructure can be obtained. Such differential will point to the locally equilibrium state of the material medium.

A degenerate transformation is a transformation that does not preserve the differential. The degenerate transformation can take place under additional conditions. These conditions can be caused by an availability of any degrees of freedom of material medium. Such conditions can be realized by the self-changing of a nonidentical relation. The vanishing of such functional expressions as determinants, Jacobians, Poisson's brackets, residues, and others corresponds to these additional conditions.

Under degenerate transformation the following transition proceeds:

$$
d \omega^{p} \neq 0 \rightarrow(\text { a degenerate transformation }) \rightarrow d_{\pi} \omega^{p}=0, d_{\pi}{ }^{*} \omega^{p}=0
$$

where the conditions $d_{\pi} \omega^{p}=0$ and $d_{\pi}{ }^{*} \omega^{p}=0$ are the conditions for the exterior and dual form closure.

Realization of the closure conditions for a dual form (metric form, which describes a pseudostructure) and realization of the closure conditions for a skewsymmetric form suggests that the pseudostructure is realized a closed dual form * $\omega_{\pi}^{p}$ and the closed inexact exterior form $\omega_{\pi}^{p}$ is formatted.

On the pseudostructure $\pi$, from evolutionary relation (3.6), we get the the relation

$$
d_{\pi} \psi=\omega_{\pi}^{p}
$$

which proves to be the identical relation, since the form $\omega_{\pi}^{p}$ is a closed one, on the pseudostructure, it turns out to be a differential. 
From an identical relation one can find the differential of the state functional $d_{\pi} \psi$. The emergence of the differential $d_{\pi} \psi$ points out to a presence of the state function. This indicates that the material medium passes into an equilibrium state. However, such a state of the material medium is realized only locally since the differential $d_{\pi} \psi$ is an internal differential (only on a pseudostructure). That is, the material medium goes into a state of local equilibrium.

(But in this case, the total state of the material medium turns out to be nonequilibrium. In this case, the total differential of the evolutionary form is nonzero. This shows that the total state of the material media remains nonequilibrium.)

The transition of the material medium at a locally-equilibrium state means that the unmeasured quantity, which acts as an internal force, converts into a measured quantity of the material medium. This results in the emergence of some observed formations in the material medium, such as waves, vortices, fluctuations, turbulent pulsations and so on.

As noted in Section 2, closed dual forms and closed inexact exterior forms made up a differential-geometric structure, which describes the physical structure, that is, a pseudostructure with a conservative quantity. Realization of such a differential-geometric structure (under degenerate transformation) points out to the emergence of a physical structure. (This will be shown in the next Section.)

Thus, from the properties of the nonidentical evolutionary relation and those of the evolutionary form one can see that under realization of the additional condition (which is a condition of degenerate transform) the transition of the material media state from nonequilibrium to locally equilibrium state can be realized. ${ }^{4}$

It was shown that the balance conservation laws regulate evolutionary processes in the material media and the transitions of the material medium from a nonequilibrium state to a locally equilibrium state. Such processes are described by solutions of equations of mathematical physics.

\subsection{Double solutions of the equations of mathematical physics}

The mathematical physics equations have a peculiarity. They have double solutions. It is the presence of double solutions that makes it possible to describe the

\footnotetext{
${ }^{4}$ This can be shown by the example of the evolutionary relation for a gas-dynamic medium [8]. The evolutionary relation for a gas-dynamic medium has the form $d s=\omega$, where $s$ is the entropy (see Appendix). If the evolutionary relation is identical, from that one would obtain the differential of entropy $d s$ and find entropy $s$ as the gas-dynamic function of state. The availability of the gas-dynamic function of state would point to the equilibrium state of the gas-dynamic medium.

But, as has been shown above, the evolutionary relation is not identical. From such a relation the differential of entropy $d s$ cannot be defined. This points out to an absence of the gas-dynamic function of state and means that the gas-dynamic medium state is nonequilibrium.

As it has been shown above, under degenerate transformation the identical relation is obtained from a nonidentical one. From an identical relation one can obtain the differential of entropy $d s$ and find entropy $s$ as the gas-dynamic function of state. The availability of the gas-dynamic function of state would point out to the equilibrium state of the gas-dynamic medium. However, since the identical relation is satisfied only under additional conditions, such a state of the gasdynamic medium will be a locally-equilibrium one.

The transition of the gas-dynamic medium at a locally equilibrium state means that an unmeasured quantity, which acts as an internal force, converts into the observed formations, such as vortices, turbulent pulsations.
} 
state of material media and transitions from a nonequilibrium state to a locally equilibrium state. This follows from the evolutionary relation obtained from the equations of the balance conservation laws.

The nonidentical evolutionary relation cannot be integrated directly since its right-hand side contains an unclosed skew-symmetric form which is not a differential. This means that the equations of mathematical physics prove to be non-integrable (they cannot be convoluted into an identical relation for differentials and be integrated). In this case, the solutions to equations of mathematical physics are not functions (their derivatives do not make up a differential). Such solutions will depend on the commutator of the evolutionary skew-symmetric form $\omega^{p}$ which is nonzero. (If the commutator is equal to zero, the evolutionary relation would be identical, and this would point out to the integrability of the original equations.)

Such solutions, which are defined on the original coordinate space, describe the nonequilibrium state of the material medium.

As shown, under the degenerate transformation following from the evolutionary form, the closed inexact (on a pseudostructure only) exterior form and the corresponding closed dual form (describing the pseudostructure) are realized.

The realization of the closed inexact exterior form and the corresponding dual form means that on pseudostructure from nonidentical evolutionary relation we have an identical relation since the closed form is a differential.

Since the identical relation can be integrated (because it contains only differentials), this means that, on a pseudostructure, the equations of mathematical physics become locally integrable only on the pseudostructure. The pseudostructure in this case is an integrable structure.

The solutions to the mathematical physics equations on integrable structures are generalized solutions, which are discrete functions since they are realized only on the integrable structures. The discrete functions correspond to the locallyequilibrium state of the material medium and describe physical structures. That is, the solutions to the mathematical physics equations possess a duality. They are obtained on original coordinate space as well as on integrable structures. A connection between these solutions and transitions from the solution on coordinate space to the solutions on integrable structures describes the mechanism of the evolutionary processes taking place in the material medium. For more details, see [11].

As shown, a transition from an evolutionary form to a closed inexact (on a pseudostructure) exterior form is described by a degenerate transformation. An example of a degenerate transform is the Legendre transformation.

The evolutionary form is defined on a tangent manifold. Closed exterior forms are defined on a cotangent (covariant) manifold. Pseudostructures are bundles of cotangent manifolds.

It turns out that, under a degenerate transformation, the transition from a tangent manifold to a cotangent manifold takes place. In this case, the realization of closed inexact exterior forms on pseudostructures indicates the emergence of physical structures on the cotangent manifold. As it was shown, this occurs discretely when the conditions of a degenerate transformation are fulfilled. 
Thus, the degenerate transformation describes the process of the emergence of physical structures on a cotangent (integrable) manifold. And a nondegenerate transformation, as is known, describes the transition on the integrable manifold from one physical structure to another. For Hamiltonian systems, this is written in $[12]$.

\section{IMPLEMENTATION OF THE EXACT CONSERVATION LAW}

As shown, closed inexact exterior forms are realized from an evolutionary form. Realization of the closed inexact exterior form, which describe the exact conservation laws, indicates the fulfillment of the exact conservation law.

The conservation laws for physical fields are exact conservation laws. As has been shown, the closed inexact exterior form (the conservative quantity) and the relevant dual form (the pseudostructure) made up a differential-geometrical structure that describes a physical structure, namely, a pseudostructure with a conservative quantity. The conservation laws for physical fields are conservation laws that state the presence of conservative quantities or objects. The physical structures are conservative objects. That is, they are subject to the conservation laws for physical fields. Massless particles, wave fronts, and so on are examples of physical structures. Physical structures and observed formations, which, as shown above, arise in material media, are not identical objects. Whereas the wave is an observable formation, the wave front is a physical structure. And, at the same time, they are a manifestation of the same phenomenon. Light is an example of a manifestation of such a duality, namely, as a massless particle, photon, and as a wave.

Since closed inexact exterior forms corresponding to a physical structure are obtained from the evolutionary relation for the material media, it follows that physical structures are generated by the material media. This is controlled by the conservation laws.

Here, one can see a connection between the balance conservation laws for the material media and the conservation laws for physical fields. The physical structures that correspond to the conservation laws for physical fields are generated by the material media based on the interaction of the noncommutative balance conservation laws. A peculiarity consists in the fact that this connection is realized discretely in the evolutionary process if there are any degrees of freedom.

It should be emphasized that the fulfillment of the exact conservation laws occurs as a result of the interaction of non-commutative balance conservation laws. This is described by the evolutionary nonidentical relation, which is obtained from the balance conservation laws, and by the identity relation, which is realized from the evolutionary relation. ${ }^{5}$

\footnotetext{
${ }^{5}$ The examples of the evolutionary nonidentical relation and the identical relation are the relations corresponding to the principles of thermodynamics [13]. The first principle of thermodynamics follows from the balance conservation laws for energy and linear momentum, and the second principle of thermodynamics follows from the first one under the fulfillment of the condition of integrability, i.e., a realization of the integrating factor (the inverse temperature). Here, it should be emphasized that the second principle of thermodynamics, unlike the first principle of thermodynamics, holds only discretely, namely, only if the integrating factor is realized.
} 
The duality of conservation laws, the connection between balance conservation laws and exact conservation law, and transitions from balance conservation laws to exact conservation law plays a regulatory role in evolutionary processes. Such properties of conservation laws reveal the causes and mechanism of the appearance of various physical structures [14] and observed formations, such as waves, vortices, fluctuations, turbulent pulsations, etc. [8], reveal the connection of the equations of field theory with the equations of mathematical physics [15], develop the physical meaning of the principles of thermodynamics [12], etc.

In this work, attention was paid to the properties of conservation laws for material media and conservation laws for physical fields. It should be noted that other systems may have properties similar to those of conservation laws for material media.

These properties are possessed by physical systems, which are described by a system of differential equations. In real processes, the differential equations forming such a system turn out to be inconsistent [11], as in the case of the equations of balance conservation laws. But in the presence of any degrees of freedom, the consistency of the equations can be realized locally. In this case, the solution to the system of equations is the differential, that is, the closed external form. This indicates the fulfillment of the exact conservation law. The inconsistency of the equations that form the system of equations and the implementation (in the evolutionary process) of the local consistency of equations reveals the mechanism of discrete transitions, quantum jumps, the emergence of various structures [11].

\section{Conclusion}

The paper has shown a connection of the conservation laws for physical fields with the conservation laws for material media.

The conservation laws for material media are conservation laws for energy, linear momentum, angular momentum, and mass. Such conservation laws are described by differential equations. They establish a balance between changes in physical quantities and external actions.

And the conservation laws for physical fields are conservation laws that state the presence of conservative physical quantities or physical objects (structures). Such conservation laws are described by closed exterior skew-symmetric forms. These are exact conservation laws.

The connection of conservation laws for physical fields with conservation laws for material media reveal the origin of physical structures corresponding to the conservation laws for physical fields, uncover the mechanism for the generation of physical structures, and the emergence of physical structures and observed formations (waves, vortices, turbulent pulsations).

The process of realizing physical structures reveals the mechanism for realizing various invariant structures. This allows us to understand the origin of existing physical objects and observed phenomena.

These results are obtained with the help of the mathematical apparatus of skew-symmetric differential forms, which possess such unconventional elements as nonidentical relations and degenerate transformations. 


\section{APPENDIX: ANALYSis of BALANCE CONSERVATION LAW EQUATIONS ON THE EXAMPlE OF THE EULER AND NAVIER-STOKES EQUATIONS GAS-DYNAMIC MEDIA. EVOLUTIONARY RELATION}

Let us consider the gas-dynamic media of ideal (non-viscous) and viscous gases that are described by Euler or Navier-Stokes equations, respectively, which simulate the conservation laws for energy, linear momentum, and mass.

Assume that the gas is a thermodynamic system in the state of local equilibrium, that is, the following relation is fulfilled [1]:

$$
T d s=d e+p d V
$$

where $T, p$ and $V$ are the temperature, the pressure, and the gas volume, $s$ and $e$ are entropy and internal energy per unit of volume. (Here iy should be emphasized that this relation describes the state of gas particles only. That is, it is assumed that the gas particles are in the equilibrium state, whereas the gasdynamic medium itself may be in the non-equilibrium state.)

Let us analyze the consistency of the equations that describe the conservation laws for energy and linear momentum [6].

In the inertial frame of reference the equation of the conservation law for energy can be written as

$$
\frac{D h}{D t}-\frac{1}{\rho} \frac{D p}{D t}=A_{1}
$$

where $D / D t$ is the total derivative with respect to time, $\rho=1 / V$ and $h$ are the density and enthalpy of the gas, respectively.

In the case of ideal gas described by the Euler equations, we have

$$
A_{1}=0
$$

and, in the case of viscous heat-conducting gas described the Navier-Stokes equations, expression $A_{1}$ can be written as

$$
A_{1}=\frac{1}{\rho} \frac{\partial}{\partial x_{i}}\left(-\frac{q_{i}}{T}\right)-\frac{q_{i}}{\rho T} \frac{\partial T}{\partial x_{i}}+\frac{\tau_{k i}}{\rho} \frac{\partial u_{i}}{\partial x_{k}} .
$$

Here, $q_{i}$ is the heat flux and $\tau_{k i}$ is the viscous stress tensor.

Expressing enthalpy in terms of internal energy $e$ with the help of formula $h=e+p / \rho$ and using relation (A1), equation (A2) of the conservation law for energy can be reduced to the form

$$
\frac{D s}{D t}=A_{1} .
$$

Since the total derivative with respect to time is that along the trajectory, in the accompanying frame of reference the equation of the conservation law for energy takes the form:

$$
\frac{\partial s}{\partial \xi^{1}}=A_{1}
$$

where $\xi^{1}$ is the coordinate along the trajectory. 
In the accompanying frame of reference, the equation of the conservation law for linear momentum can be presented as

$$
\frac{\partial s}{\partial \xi^{\nu}}=A_{\nu}
$$

where $\xi^{\nu}$ is the coordinate in the direction normal to the trajectory.

In the case of two-dimensional ideal gas, from the Euler and Navier-Stokes equations, one can obtain the following expression for coefficient $A_{\nu}$ :

where $\zeta=\partial u_{2} / \partial x-\partial u_{1} / \partial y$.

$$
A_{\nu}=\frac{\partial h_{0}}{\partial \nu}+\left(u_{1}^{2}+u_{2}^{2}\right)^{1 / 2} \zeta-F_{\nu}+\frac{\partial U_{\nu}}{\partial t}
$$

In the case of viscous gas, the expression $A_{\nu}$ includes additional terms related to viscosity and heat-conductivity. Equations (A3) and (A4) can be convoluted into the relation

$$
d s=\omega
$$

where $\omega=A_{\mu} d \xi^{\mu}$ is the first-degree skew-symmetric differential form and $\mu=$ $1, \nu$. Since the equations of conservation laws are evolutionary ones, the relation obtained is also an evolutionary relation. In this case, the skew-symmetric form $\omega$ is also an evolutionary one.

\section{REFERENCES}

[1] R.W. Haywood, Equilibrium Thermodynamics for Engineers and Scientists, WileyBlackwell, 1980.

[2] É. Cartan, Les Systèmes Differentials Extérieus ef Leurs Application Géométriques, Hermann \& cie, Paris, 1945.

[3] L. I. Petrova, Skew-symmetric Differential Forms. Conservation Laws. The Foundation of Equations of Mathematical Physics and Field Theory, Krasand URSS, Moscow, 2020.

[4] L. I. Petrova, Exterior and evolutionary skew-symmetric differential forms and their role in mathematical physics, http://arxiv.org/pdf/math-ph/0310050v1.pdf, 2003.

[5] R. Bott, L. W. Tu, Differential Forms in Algebraic Topology, Springer, NY, 1982.

[6] J. F. Clark, M. Machesney, The Dynamics of Real Gases, Butterworths, London, 1964.

[7] R. C. Tolman, Relativity, Thermodynamics, and Cosmology, Clarendon Press, Oxford, UK, 1969.

[8] L. I. Petrova, Features of Numerical Simulation of Euler and Navier-Stokes Equations, Comput. Math. Model. 28 (2017), 32-36.

[9] L. I. Petrova, Physical meaning and a duality of concepts of wave function, action functional, entropy, the pointing vector, the Einstein tensor, J. Math. Res. 4 (2012), 78-88.

[10] M.-A. Tonnelat, Les principles de la théorie électromagnétique et de la relativité, Masson, Paris, 1959.

[11] L. Petrova, Discrete quantum transitions, duality: Emergence of physical structures and occurrence of observed formations (hidden properties of mathematical physics equations), Journal of Applied Mathematics and Physics (SciRP) 8 (2020), 1911-1921.

[12] L. Petrova, Qualitative investigation of Hamiltonian systems by application of skewsymmetric differential forms, Symmetry 13 (2021), 7 pp.

[13] L. I. Petrova, Connection between the principles of thermodynamics and the conservation laws: Physical meaning of the principles of thermodynamics, Journal of Applied Mathematics and Physics (SciRP) 6 (2018), 2697-2704.

[14] L. I. Petrova, Formatting physical fields and pseudometric manifolds. The dark matter, J. Phys.: Conf. Ser. 1557 (2020), Paper No. 012032, 8 pp. 
[15] L. Petrova, Evolutionary relation of mathematical physics equations evolutionary relation as foundation of field theory interpretation of the Einstein equation, Axioms 10 (2021), $10 \mathrm{pp}$.

Ludmila I. Petrova, Moscow State University, Department of Computational Mathematics and Cybernetics, Moscow, Russia

e-mail: ptr@cs.msu.ru 